\title{
ICAR 2019 Special Issue
}

\author{
Guilherme A. S. Pereira ${ }^{1}$ (D) . Paulo L. J. Drews Jr. ${ }^{2}$ (D) - Denis F. Wolf ${ }^{3}$ (D) - Leonardo S. Mattos ${ }^{4}$ (D)
}

Received: 13 July 2021 / Accepted: 14 July 2021 / Published online: 23 July 2021

(C) The Author(s), under exclusive licence to Springer Nature B.V. 2021

The $19^{\text {th }}$ International Conference on Advanced Robotics (ICAR) took place in the beautiful city of Belo Horizonte, Brazil, hosted by the Federal University of Minas Gerais (UFMG). In this edition, ICAR, which is one of the oldest and most prestigious robotics conferences in the world, was chaired by Prof. Mario Campos, from UFMG, and co-chaired by Prof. Paolo Fiorini, from University of Verona, in Italy. From December $2^{\text {nd }}$ to December $4^{\text {th }}$ of 2019, 124 high quality research papers in the several areas of robotics were presented orally in four parallel technical sections. All the papers presented in the conference can be found in the IEEE Explore. ${ }^{1}$ Among those papers, the best ones were invited to be extended, while keeping a similarity score smaller than $30 \%$, to be considered for this special issue. Fifty (50) papers were submitted and, after a careful review process, 22 papers were finally accepted for publication in the Journal of Intelligent \& Robotic Systems.

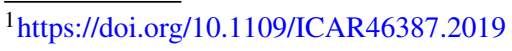

Guilherme A. S. Pereira

guilherme.pereira@mail.wvu.edu

Paulo L. J. Drews Jr.

paulodrews@furg.br

Denis F. Wolf

denis@icmc.usp.br

Leonardo S. Mattos

leonardo.demattos@iit.it

1 Department of Mechanical and Aerospace Engineering, West Virginia University, Morgantown, WV, USA

2 Center for Computational Science, Federal University of Rio Grande, Rio Grande, RS, Brazil

3 Institute of Mathematics and Computer Sciences, University of São Paulo, Butanta, SP, Brazil

4 Department of Advanced Robotics, Istituto Italiano di Tecnologia, Genoa, Italy
The diversity of areas of ICAR was reflected in this special issue. Among the published papers, it is possible to find robotic applications that range from urban mobility to underground exploration and medical robotics. Those papers present theoretical advances in areas that include robot motion planning and obstacle avoidance, navigation, teleoperation, perception, system identification, non-linear control, human-robot collaboration, intelligent control, multi-robot systems, and robotic swarms. The special issue was published as a topical collection with the following papers:

- Azpúrua, H., Rezende, A., Potje, G. et al. Towards Semi-autonomous Robotic Inspection and Mapping in Confined Spaces with the EspeleoRobô.

- Chandarana, M., Hughes, D., Lewis, M. et al. Planning and Monitoring Multi-Job Type Swarm Search and Service Missions.

- Coelho, A., Sarkisov, Y., Wu, X. et al. Whole-Body Teleoperation and Shared Control of Redundant Robots with Applications to Aerial Manipulation.

- Fonseca, M.d.P.A., Adorno, B.V. \& Fraisse, P. TaskSpace Admittance Controller with Adaptive Inertia Matrix Conditioning.

- Franco, I.J.P.B., Ribeiro, T.T. \& Conceição, A.G.S. A Novel Visual Lane Line Detection System for a NMPC-based Path Following Control Scheme.

- Garcia, G., Rocha, F., Torre, M. et al. ROSI: A robotic system for harsh outdoor industrial inspection.

- Ginesi, M., Meli, D., Roberti, A. et al. Dynamic Movement Primitives: Volumetric Obstacle Avoidance Using Dynamic Potential Functions.

- Glogowski, P., Böhmer, A., Hypki, A. et al. Robot Speed Adaption in Multiple Trajectory Planning and Integration in a Simulation Tool for Human-Robot Interaction.

- Gomes, I.P., Wolf, D.F. Health Monitoring System for Autonomous Vehicles using Dynamic Bayesian Networks for Diagnosis and Prognosis.

- de Jesus, J., Kich, V.A., Kolling, A.H. et al. Soft Actor-Critic for Navigation of Mobile Robots. 
- $\quad$ Kaden, S., Thomas, U. Optimizing Mobility of Robotic Arms in Collision-free Motion Planning.

- Mahé, A., Richard, A., Aravecchia, S. et al. Evaluation of Prioritized Deep System Identification on a Path Following Task.

- Naceri, A., Mazzanti, D., Bimbo, J. et al. The Vicarios Virtual Reality Interface for Remote Robotic Teleoperation.

- Nottensteiner, K., Sachtler, A. \& Albu-Schäffer, A. Towards Autonomous Robotic Assembly: Using Combined Visual and Tactile Sensing for Adaptive Task Execution.

- Oliveira, F.G., Neto, A.A., Howard, D. et al. ThreeDimensional Mapping with Augmented Navigation Cost through Deep Learning.

- Pereira, J.C., Leite, V.J.S. \& Raffo, G.V. Nonlinear Model Predictive Control on SE(3) for Quadrotor Aggressive Maneuvers.

- Pinto, M.L., Wehrmeister, M.A. \& de Oliveira, A.S. Real-Time Performance Evaluation for Robotics.

- Pires, A.G., Rezeck, P.A.F., Chaves, R.A. et al. Cooperative Localization and Mapping with Robotic Swarms.

- Sánchez, I., D’Jorge, A., Raffo, G.V. et al. Nonlinear Model Predictive Path Following Controller with Obstacle Avoidance.

- Simonič, M., Petrič, T., Ude, A. et al. Analysis of Methods for Incremental Policy Refinement by Kinesthetic Guidance.
- Soares, J.C.V., Gattass, M. \& Meggiolaro, M.A. CrowdSLAM: Visual SLAM Towards Crowded Environments using Object Detection.

- Srisuchinnawong, A., Wang, B., Shao, D. et al. Modular Neural Control for Gait Adaptation and Obstacle Avoidance of a Tailless Gecko Robot.

The guest editors would like to thank the program committee members and reviewers of ICAR 2019 for selecting the best candidate papers for this special issue. We also thank the authors of the selected papers for their effort in extending their manuscripts and submitting their work for review. We also offer our gratitude to the journal reviewers. The outcome of their hard work during a long pandemic resulted in a very strong special issue that, we hope, will also be appreciated by the robotics community. Finally, we thank the Editor-in-Chief, Prof. Kimon P. Valavanis, and the Journal's Editorial Office assistant, Marie Veth Chua, for their amazing support.

The Guest Editors

Guilherme A. S. Pereira

Paulo L. J. Drews Jr.

Denis F. Wolf

Leonardo S. Mattos

Publisher's Note Springer Nature remains neutral with regard to jurisdictional claims in published maps and institutional affiliations. 